\title{
Evaluation of subjective symptoms of Japanese patients with multiple chemical sensitivity using QEESI $^{\odot}$
}

\author{
Sachiko Hojo $\cdot$ Kou Sakabe $\cdot$ Satoshi Ishikawa $\cdot$ \\ Mikio Miyata $\cdot$ Hiroaki Kumano
}

Received: 3 March 2008/Accepted: 9 June 2009/Published online: 15 July 2009

(c) The Japanese Society for Hygiene 2009

\begin{abstract}
Objectives The Quick Environment Exposure Sensitivity Inventory $\left(\mathrm{QEESI}^{\odot}\right)$ has been used as a questionnaire to evaluate subjective symptoms of patients with multiple chemical sensitivity (MCS), also known as idiopathic environmental intolerance, in Japan. However, no cutoff value for Japanese subjects has yet been established. We designed this study to establish a cutoff value for Japanese subjects using QEESI $^{\odot}$ for screening of MCS patients.

Methods A questionnaire using the QEESI $^{\odot}$ was administered to 103 MCS patients and 309 healthy control subjects matched for age and sex. QEESI ${ }^{\odot}$ scores of the two groups were compared using logistic regression analysis, receiver operating characteristic analysis, and the Mann-Whitney test.

Results Cutoff values for Japanese subjects were determined for the Chemical Intolerance subscale (40), Symptom Severity subscale (20), and Life Impact subscale (10).
\end{abstract}

S. Hojo ( $\square)$

Department of Environmental Science,

Shokei Gakuin University, 4-10-1 Yurigaoka,

Natori, Miyagi 981-1295, Japan

e-mail: hojo@shokei.ac.jp

K. Sakabe

Department of Human Structure and Function,

Tokai University School of Medicine,

Kanagawa, Japan

K. Sakabe $\cdot$ S. Ishikawa $\cdot$ M. Miyata

Division of Environmental Medical Center,

Kitasato Institute Hospital, Tokyo, Japan

H. Kumano

Faculty of Human Sciences, Waseda University,

Saitama, Japan
The subjects whose scores exceeded the cutoff values in any two subscales accounted for $88.4 \%$ of the patients but only $14.5 \%$ of the controls.

Conclusions Our results suggest that subjects meeting two out of three subscale criteria can be screened as "patients suffering from a low level of environmental chemicals such as MCS" in Japan.

Keywords Multiple chemical sensitivity (MCS) . Idiopathic environmental intolerance (IEI) .

Quick Environment Exposure Sensitivity Inventory $\left(\right.$ QEESI $\left.^{\odot}\right) \cdot$ Logistic regression analysis .

Receiver operating characteristics (ROC) analysis

\section{Introduction}

Multiple chemical sensitivity (MCS) is the term most commonly used to describe a disorder which is characterized by a broad array of physical, psychological, and emotional symptoms, the cause of which is attributed to exposure to extremely low levels of a wide variety of environmental chemicals [1]. MCS, also known as idiopathic environmental intolerance (IEI), is a syndrome which progresses to multiple organ dysfunction, mainly involving the autonomic nervous system, with extremely low-level chemical exposure [2-10]. However, the pathogenesis and underlying mechanisms have not been fully clarified anywhere in the world, and internationally unified diagnostic criteria for MCS have not been established [1]. At present, there are at least seven case definitions for MCS, including those of Randolf [11], Cullen [12], Nethercott [13], and the 1999 Consensus in the USA [14], but no case definition has been proven worldwide. Among them, the 1999 Consensus is considered the most 
comprehensive and well-known case definition, and is now being used in the USA, Germany, and a number of other countries [15]. In Japan, however, the only diagnostic standard currently in use for MCS is the diagnostic guideline proposed in 1998 by the Allergy Research Study Project of the Ministry of Health, Labor, and Welfare [16]. Various objections have been raised to each of these different standards, however, and there is still much room for further debate on this issue.

Given this situation, we considered that the most effective way to establish a reliable diagnostic standard for MCS in Japan would be to identify the characteristic features of Japanese MCS patients, and then to undertake a detailed analysis of a group of these patients selected according to a diagnostic standard that would allow comparison with results from other countries.

In the USA, Miller and Prihoda [17, 18] developed a globally standardized self-administered questionnaire, the Quick Environment Exposure Sensitivity Inventory $\left(\right.$ QEESI $\left.^{\circledR}\right)$, which was designed to assist researchers and clinicians in screening, studying, and evaluating patients for MCS. QEESI ${ }^{\odot}$ is a questionnaire recommended in the 1999 Consensus mentioned above. The Japanese version of QEESI $^{\odot}$ was translated by Ishikawa and Miyata [19]. We [20] surveyed the reliability and validity of the QEESI $^{\odot}$ (Japanese version) in the Japanese population and concluded that 30 questions of three subscales in the QEESI ${ }^{\odot}$ (Chemical Intolerance, Symptom Severity, and Life Impact) showed high reliability and validity that allowed comparison between MCS patients in Japan and those in other countries. However, Other Intolerance, the fourth QEESI $^{\odot}$ subscale, showed small factor loading with random scattering. Therefore, we concluded that this subscale is not useful in Japan and that revision of this subscale is thus required for more accurate assessment of Japanese patients [20].

Additionally, we [21] conducted a questionnaire survey using the QEESI ${ }^{\odot}$ (Japanese version) in 498 Japanese subjects not previously diagnosed as having MCS, and 17 subjects were consequently identified as having clinical features consistent with "very suggestive of MCS" based on the criteria of Miller and Prihoda (Chemical Intolerance $\geq 40$ and Symptom Severity $\geq 40$ ) [17]. In accordance with our recommendation, 7 of the 17 subjects participated in a medical examination and were diagnosed as having MCS by expert physicians of the Environmental Medical Center, Kitasato Institute Hospital, suggesting the QEESI ${ }^{\odot}$ (Japanese version) to be useful for screening MCS patients in Japan [21]. However, we [22] also compared QEESI ${ }^{\odot}$ scores between Japanese MCS patients and the American MCS patients reported by Miller and Prihoda [17]. We found the scores of the Japanese MCS patients to be considerably lower than those of the American MCS patients, indicating that a unique Japanese cutoff value is necessary to screen Japanese MCS patients.

Therefore, we designed this study to establish a cutoff value for Japanese subjects using QEESI ${ }^{\circledR}$ for the screening of MCS patients.

\section{Methods}

Subjects

\section{MCS patient group}

The subjects were 103 patients ( 24 males with mean age of $36.2 \pm 9.9$ years and 79 females with mean age of $42.9 \pm 13.8$ years) who visited the Outpatient Division of the Environmental Medical Center, Kitasato Institute Hospital during the 3-year period from March 2001 to March 2004. They met the 1999 Consensus (US) [14] and the Japanese diagnostic criteria for MCS [16], had been diagnosed as having MCS by three specialists, and had also completed the QEESI ${ }^{\circledR}$ questionnaire. The symptoms associated with MCS have similarities to chronic fatigue syndrome (CFS), fibromyalgia (FM), and psychological disorders [1]. Therefore, patients who had been diagnosed by a doctor as having chronic fatigue syndrome or fibromyalgia syndrome were excluded from the study. In addition, patients suspected of having psychological disorders were examined by a qualified psychiatrist or practitioner of psychosomatic medicine, and those patients diagnosed with mental health disorders based on DSM-III or ICD-10 were also excluded from the study.

\section{Control group}

The controls were 309 healthy people matched for age and sex with the MCS patient group (72 males and 227 females). We sent a QEESI $^{\circledR}$ (Japanese version) and a general health questionnaire [asking about current health status (good/bad), whether the subject had been diagnosed with a disease by a physician, and if so, the name of the disease, etc.], to 4,000 members of the general public who belonged to universities, architect groups, and cooperative societies in 26 prefectures in Japan, including Hokkaido, Miyagi, Kanagawa, Tochigi, Saitama, Tokyo, Nagano, Oita, and Aichi, from April 2003 to March 2005.

A total of 2,564 responses were received, and after excluding incomplete responses, 2,500 were determined to be valid. Subjects who reported by themselves that they were unhealthy or had been diagnosed with any disease by a doctor [MCS, sick house syndrome (SHS), diabetes, mental disorders including depression, etc.] were further 
excluded. A sex- and age-matched ( \pm 5 years; $N=309$ ) control group was randomly selected from the remaining 1,857 subjects.

Objective clinical examinations for MCS patients

All 103 MCS patients underwent the three neuro-ophthalmologic examinations (smooth pursuit eye movement, contrast sensitivity function, and pupillary reaction to light) included in the Japanese MCS diagnostic criteria, as well as general neurological and electrocardiographic examinations described previously [21, 23]. Each of these tests is used in the diagnosis of diseases other than MCS. The normal range was determined by evaluating data from a large number of healthy Japanese, and those whose results fell outside the normal range were diagnosed as abnormal $[21,23]$.

Questionnaire used in this study

In this study we used 30 items in three subscales (Chemical Intolerance, Symptom Severity, and Life Impact) of QEESI $^{\odot}$ outlined below (Table 1). The MCS patients and control subjects had completed the QEESI ${ }^{\odot}$.

\section{Chemical Intolerance scale}

This scale was comprised of ten common chemical inhalants that represent structurally diverse classes of chemicals to which MCS patients frequently attribute their symptoms [24]: diesel or gas engine exhaust (petrochemical combustion products), tobacco smoke, insecticide (pesticides), gasoline vapors (fuels), paints or paint thinner (solvents), cleaning products such as disinfectants or bleach (structurally diverse clearing agents), fragrances, fresh tar or asphalt (polynuclear aromatics), nail polish, nail polish remover or hairspray, new furnishings such as carpets (fragrance/solvent), and soft plastic shower curtains or the interior of new cars (solvents, plasticizers, formaldehyde). Each item includes a $0-10$ rating scale, the ratings being tallied to obtain a total scale score of $0-100$. Miller and Prihoda [16] defined the criteria for three levels of symptom score as low (0-19), medium (20-39), and high (40100).

\section{Symptom Severity scale}

The ten items on this scale were derived via factor analysis of responses to 114 symptoms used in a study of 112 IEI patients who were exposed to either pesticides or indoor contaminants [24]. Items include musculoskeletal, airway/ mucous membrane, heart/chest-related, gastrointestinal, cognitive, affective, neuromuscular, head-related, skin, genitourinary, and symptoms. Each item includes a 0-10 rating scale, summed to obtain a total score of $0-100$. Miller and Prihoda [16] defined the criteria for three levels of symptom score as low (0-19), medium (20-39), and high (40-100).

\section{Life Impact scale}

Respondents rated the degree to which chemical or food sensitivities adversely impacted each of ten aspects of their lives: diet, ability to work or attend school, choice of home furnishings, choice of clothing, ability to drive or travel, choice of personal care products such as cosmetics or deodorants, ability to be around others and enjoy social activities, choice of hobbies or recreation, relationships with spouse and family, and ability to perform household chores. Each item includes a 0-10 rating scale, summed to obtain a total score of $0-100$. Miller and Prihoda [17] defined the criteria for three levels of symptom score as low (0-11), medium (12-23), and high (24-100).

\section{Statistical analysis}

In order to statistically analyze subjective symptoms of the MCS patient group and the control group, we compared the QEESI $^{\odot}$ scores of 30 items in three subscales (Symptom Severity, Chemical Intolerance and Life Impact) by logistic regression analysis and receiver operating characteristics (ROC) analysis using SPSS version 13 for Windows.

\section{Ethical considerations}

This study was approved by the Human Ethics Committee of the Environmental Medical Center of Kitasato Institute Hospital, and all participants gave written informed consent. No individual names or other data allowing identification of participants were included in the database.

\section{Results}

Frequency distribution of QEESI $^{\circledR}$ scores

Figure 1 shows the frequency distribution of the total points for three subscales in the QEESI $^{\odot}$ (Symptom Severity, Chemical Intolerance, and Life Impact). While the control group showed an ever-decreasing distribution, with the highest frequency being that of 0 in all three subscales, the MCS patient group showed a widely scattered distribution. However, high scores, 40 points or more, for Chemical Intolerance were relatively common in the control group as well. 
Table 1 Logistic regression analysis of three subscales in QEESI $^{\odot}$
Exp $(B)$ : odds ratio. Individual items in each subscale score of $0-10$. Total score of each item is $0-100$

\begin{tabular}{|c|c|c|c|c|}
\hline \multirow[t]{2}{*}{ Items } & & \multirow[t]{2}{*}{$\operatorname{Exp}(B)$} & \multicolumn{2}{|c|}{$95 \%$ confidence interval } \\
\hline & & & Min. & Max. \\
\hline \multicolumn{5}{|c|}{ Q1 Chemical Intolerance } \\
\hline q1_9 & Nail polish or hairspray & 1.57 & 1.41 & 1.74 \\
\hline q1_10 & New furnishings & 1.56 & 1.41 & 1.73 \\
\hline q1_7 & Fragrances & 1.52 & 1.38 & 1.67 \\
\hline q1_6 & Cleaning products & 1.51 & 1.37 & 1.67 \\
\hline q1_5 & Paint or paint thinner & 1.43 & 1.31 & 1.57 \\
\hline q1_3 & Insecticide & 1.43 & 1.30 & 1.56 \\
\hline q1_4 & Gasoline & 1.36 & 1.24 & 1.50 \\
\hline q1_1 & Diesel or gasoline exhaust & 1.33 & 1.21 & 1.46 \\
\hline q1_2 & Tobacco smoke & 1.33 & 1.22 & 1.45 \\
\hline \multirow[t]{2}{*}{ q1_8 } & Tar or asphalt & 1.32 & 1.20 & 1.44 \\
\hline & Q1 total & 1.05 & 1.04 & 1.07 \\
\hline \multicolumn{5}{|c|}{ Q3 Symptom Severity } \\
\hline q3_5 & Cognitive & 1.95 & 1.71 & 2.22 \\
\hline q3_2 & Airway/mucous membranes & 1.90 & 1.68 & 2.15 \\
\hline q3_3 & Heart/chest-related & 1.88 & 1.65 & 2.15 \\
\hline q3_8 & Head-related & 1.86 & 1.64 & 2.10 \\
\hline q3_7 & Neuromuscular & 1.84 & 1.63 & 2.08 \\
\hline q3_6 & Affective & 1.75 & 1.56 & 1.96 \\
\hline q3_1 & Musculoskeletal & 1.59 & 1.44 & 1.77 \\
\hline q3_4 & Gastrointestinal & 1.57 & 1.43 & 1.73 \\
\hline q3_10 & Genitourinary & 1.56 & 1.40 & 1.74 \\
\hline \multirow[t]{2}{*}{ q3_9 } & Skin & 1.46 & 1.33 & 1.60 \\
\hline & Q3 total & 1.15 & 1.12 & 1.18 \\
\hline \multicolumn{5}{|c|}{ Q5 Life Impact } \\
\hline q5_2 & Ability to work or attend school & 2.74 & 2.20 & 3.43 \\
\hline q5_7 & Ability to be around others and enjoy social activities & 2.66 & 2.06 & 3.43 \\
\hline q5_8 & Choice of hobbies or recreation & 2.31 & 1.88 & 2.85 \\
\hline q5_3 & Choice of home furnishings & 2.03 & 1.73 & 2.38 \\
\hline q5_1 & Diet & 1.92 & 1.57 & 2.34 \\
\hline q5_10 & Ability to perform household chores & 1.86 & 1.61 & 2.15 \\
\hline q5_9 & Relationships with spouse and family & 1.79 & 1.52 & 2.10 \\
\hline q5_6 & Choice of personal care products & 1.70 & 1.52 & 1.91 \\
\hline q5_5 & Ability to drive or travel & 1.55 & 1.40 & 1.71 \\
\hline \multirow[t]{2}{*}{ q5_4 } & Choice of clothing & 1.51 & 1.36 & 1.69 \\
\hline & Q5 total & 1.18 & 1.14 & 1.23 \\
\hline
\end{tabular}

\section{Setting of a cutoff value}

We performed ROC analysis to briefly screen the MCS patients using the QEESI $^{\odot}$ (Fig. 2). The cutoff value was set when the total for sensitivity and specificity reached the maximum, i.e., 40 points for Chemical Intolerance, 20 for Symptom Severity, and 10 for Life Impact. Symptom Severity had the highest discrimination ability, showing a sensitivity of $84.8 \%$, specificity of $84.0 \%$, and ROC area under the curve of 0.935 . Life Impact also had high discrimination ability, showing a sensitivity of $84.8 \%$, specificity of $85.7 \%$, and ROC area under the curve of 0.928. However, for the Chemical Intolerance subscale, sensitivity, specificity, and ROC area under the curve had low discrimination abilities (73.4, 69.6, and $0.779 \%$, respectively) compared with the other two subscales.

\section{Classification using cutoff values}

Figure 3 shows the distribution of subjects in the MCS patient group and the control group by classification using the cutoff values provided above. The number of subjects 


\section{MCS patients}

Chemical Intolerance

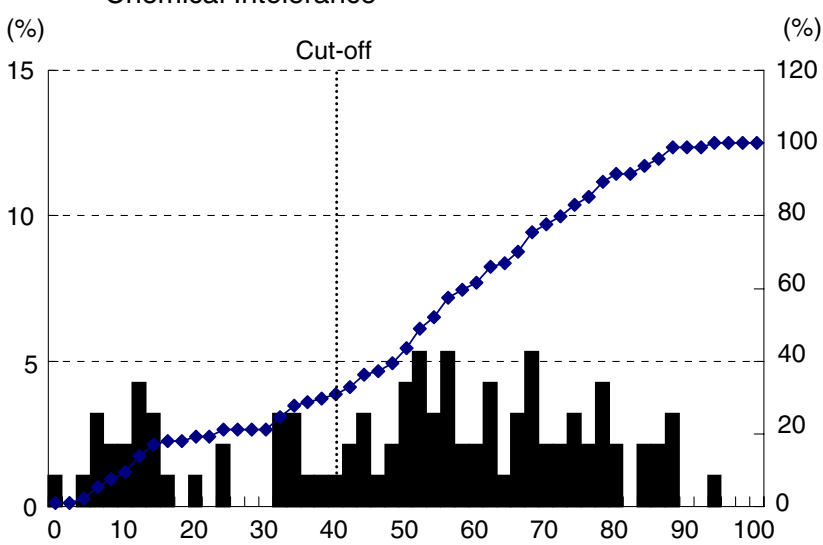

Symptom Severity

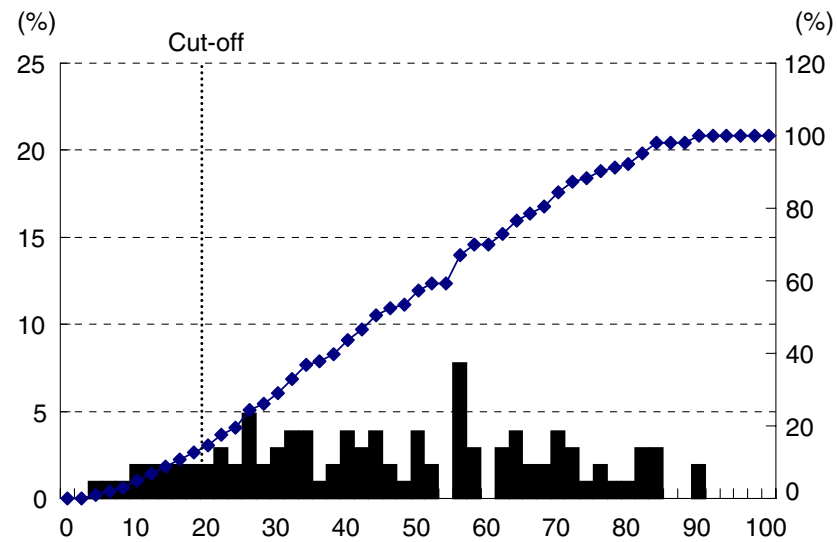

Life Impact

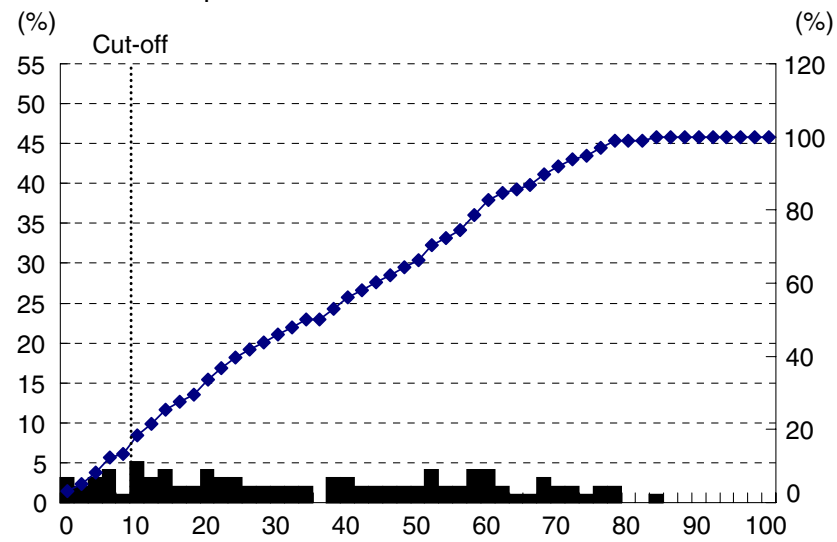

$\%)$

\section{Controls}

(\%) (\%)

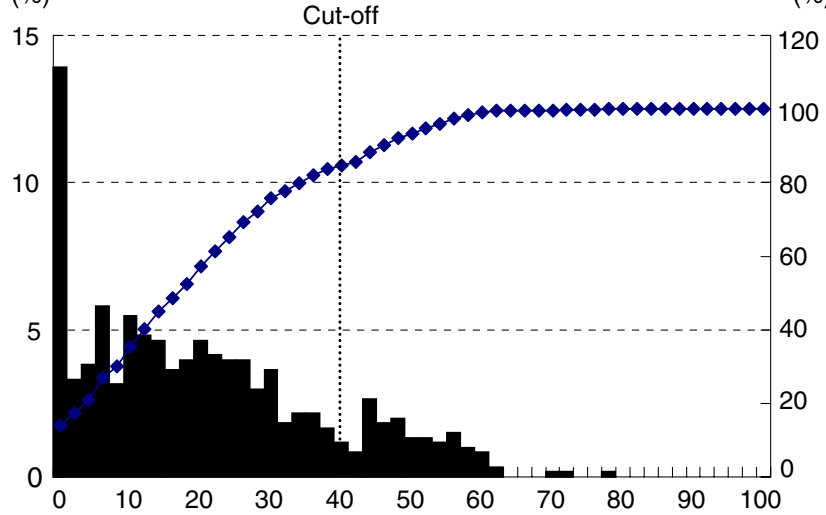

(\%) $\quad$ (\%)

25 Cut-off $\ldots \ldots \ldots \ldots$
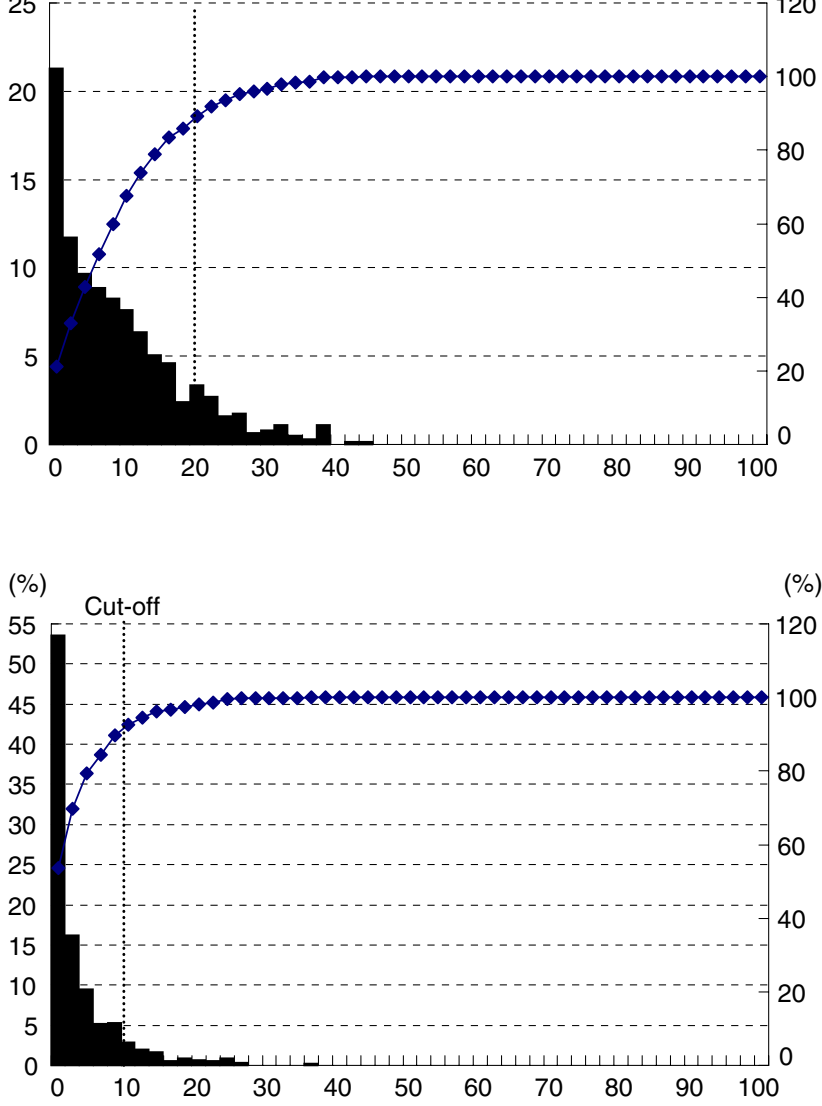

(\%)

120

100

80

60

40

20

100

Fig. 1 Frequency distributions of the total points of three subscales in the QEESI ${ }^{\odot}$ (Chemical Intolerance, Symptom Severity, and Life Impact) for MCS patients (103) and controls (309)

for whom the three circles at Chemical Intolerance $\geq 40$, Symptom Severity $\geq 20$, and Life Impact $\geq 10$ overlapped was $62(60.2 \%)$ in the MCS patient group and $10(3.2 \%)$ in the control group. Furthermore, $88.4 \%$ of the MCS patient group, but only $14.5 \%$ of the control group, reached or exceeded the cutoff value in any two of the three subscales (Fig. 3).
Radar chart of the three subscales in QEESI $^{\circledR}$

Mean scores in all 30 items for the MCS patients were significantly higher than those for controls (Fig. 4a-c). However, there were no differences in the radar chart patterns of Chemical Intolerance and Symptom Severity between the MCS patients and the controls. The radar chart 
pattern of the Life Impact subscale differed somewhat between the two groups. The peaks were in "ability to work," "choice of home furnishings," "choice of personal

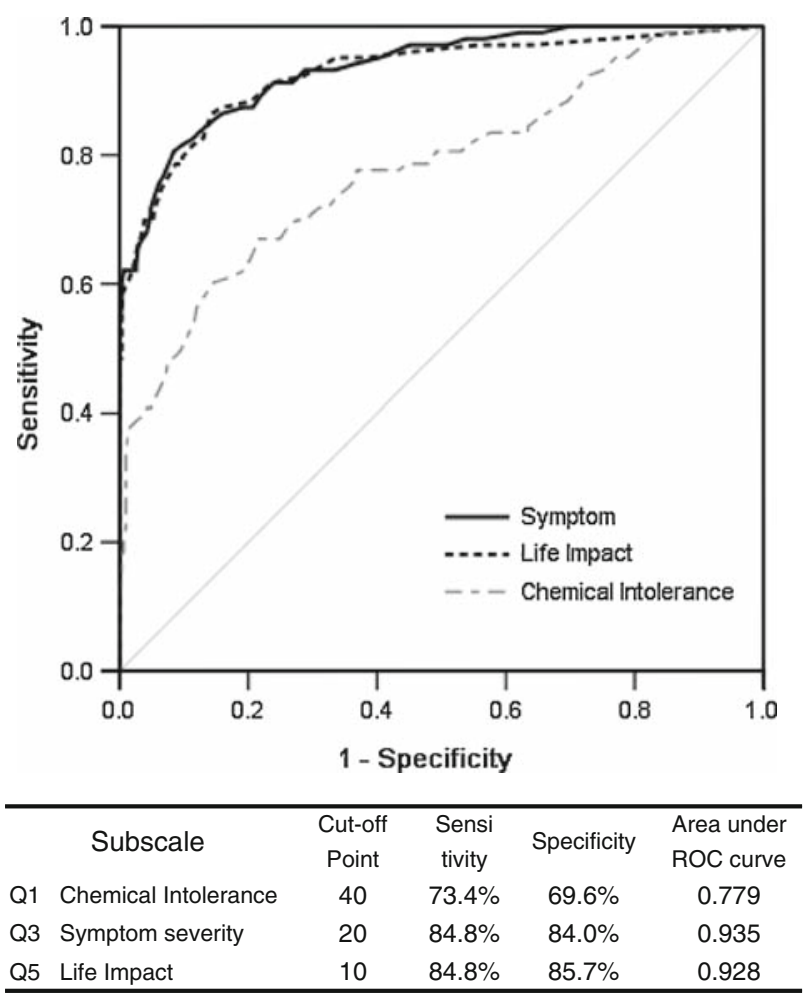

Fig. 2 Results of receiver operating characteristics analysis care products," and "social activity," while the scores for "diet" and "relationships with spouse and family" were relatively low in the MCS patient group.

Logistic regression analysis of 30 items in three subscales

In order to study the discrimination abilities of all subjective symptoms, we performed logistic regression analysis for a total of 30 items in the three subscales of Chemical Intolerance, Symptom Severity, and Life Impact (Table 1).

\section{Chemical Intolerance}

The odds ratio was 1.32-1.57. The differences among individual items were small. The odds ratios, in descending order, were as follows: "nail polish or hairspray" (1.57) > "new furnishings" (1.56) > "fragrances" $(1.52)>$ "cleaning products" (1.51) > "paint or paint thinner" (1.43).

\section{Symptom Severity}

The odds ratio was 1.46-1.95 and exceeded 1.5, except for "skin" (1.46). In particular, the odds ratios for the following items were more than 1.7 , showing high discrimination ability: "cognitive" (1.95) > "airway/mucous membranes" $(1.90)>$ "heart/chest-related" (1.88) > "head-related" (1.86) > "neuromuscular" (1.84) > "affective" (1.75).
Fig. 3 Distribution of subjects in the MCS patient group and the control group by classification using the cutoff values of three subscales in QEESI $^{\odot}$ (Chemical Intolerance, Symptom Severity, and Life Impact)

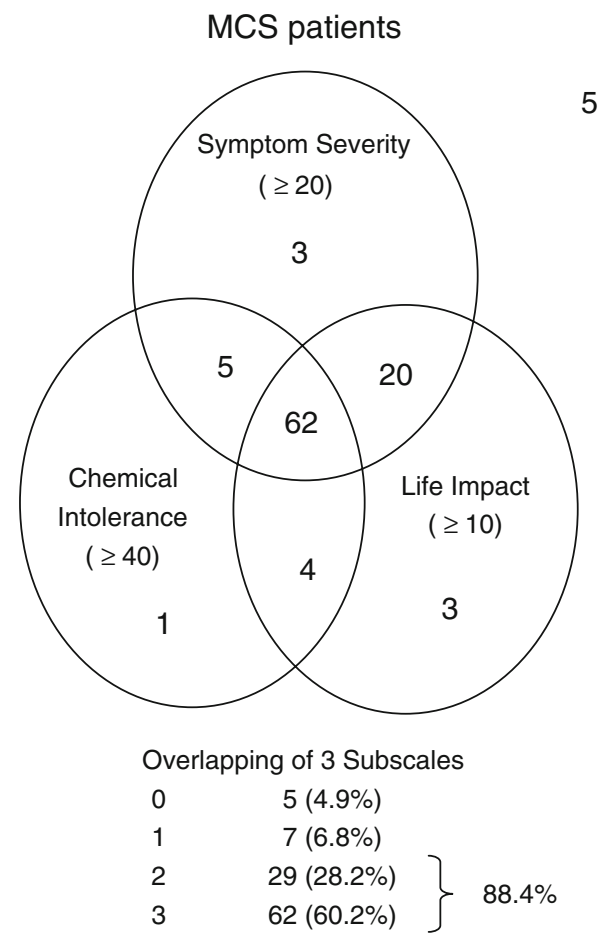

5

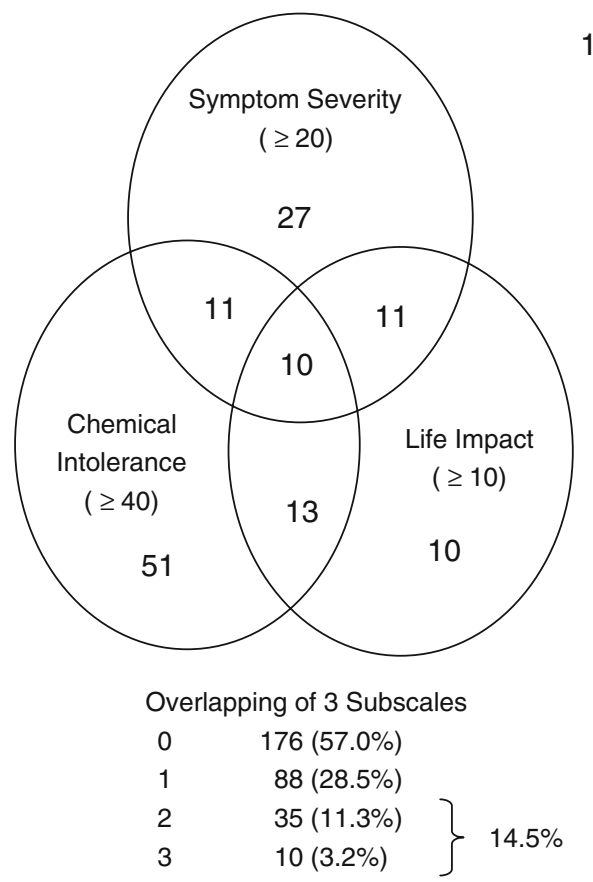


a Chemical Intolerance

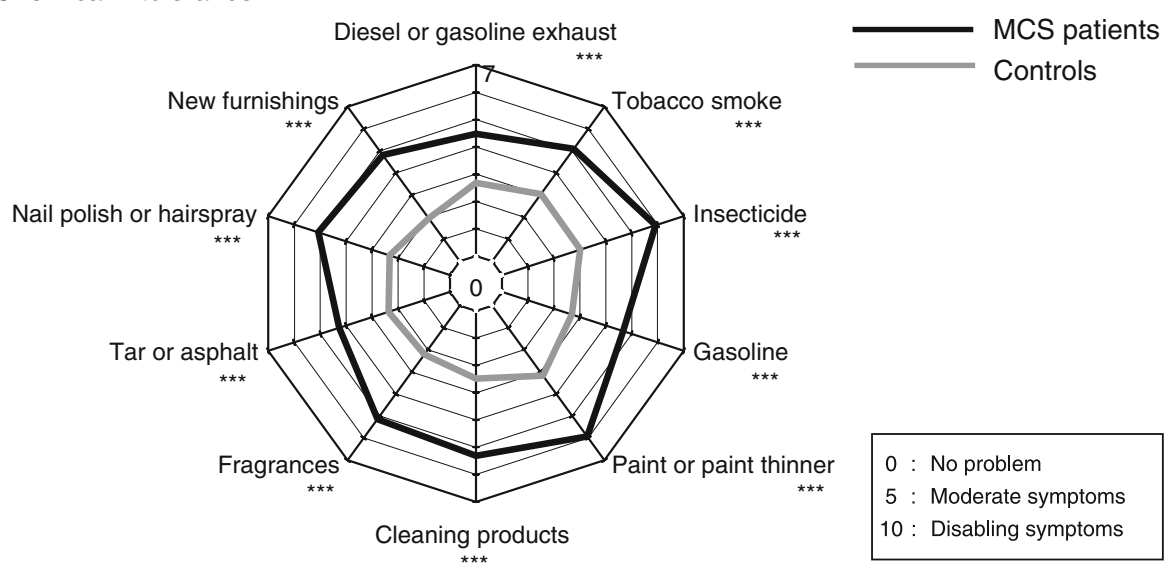

b Symptom Severity

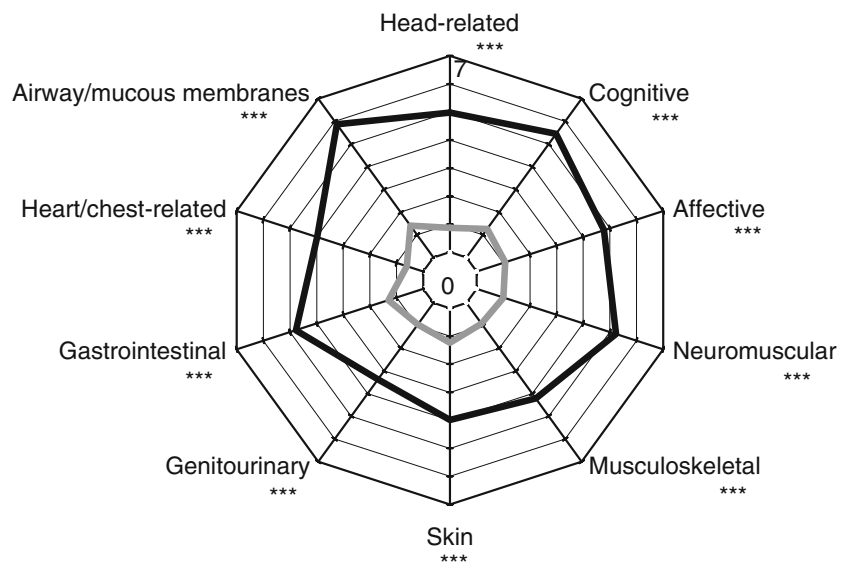

0 : No problem

10: Disabling symptoms

\section{Life Impact}

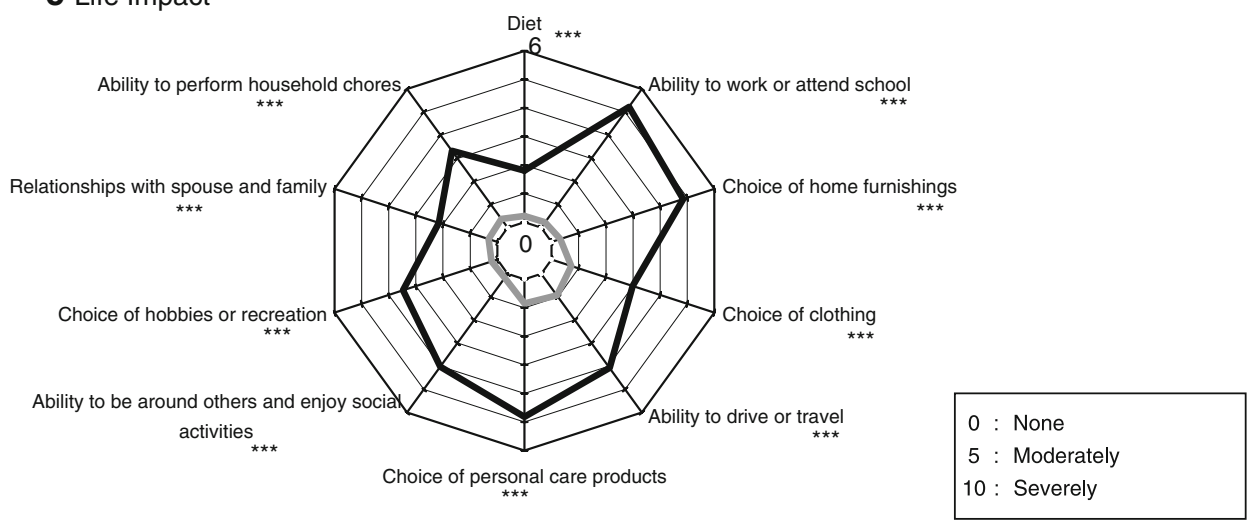

Fig. 4 Comparison of mean scores of 30 items in three subscales in QEESI ${ }^{\odot}$ (Chemical Intolerance, Symptom Severity, and Life Impact) between MCS patients and controls. ${ }^{*} * * P<0.001, * P<0.05$

\section{Life Impact}

The odds ratio was 1.51-2.74, the highest of the three subscales. In particular, the odds ratio was 1.8 or more for "ability to work or attend school" (2.74) > "ability to be around others and enjoy social activities" (2.66) > "choice of hobbies or recreation" (2.31) $>$ "choice of home furnishings" (2.03) > "diet" (1.92) > "ability to perform household chores" (1.86). Furthermore, the odds ratio was high for MCS-specific impairment, which was not seen in 
other diseases, including "choice of home furnishings" (2.03) and "choice of personal care products" (1.70).

\section{Discussion}

In this study, we performed a questionnaire survey using the QEESI $^{\circledR}$ in clinically diagnosed patients with MCS, also known as IEI, and healthy individuals matched for age and sex, in order to set a unique cutoff value for screening of MCS patients in Japan. We determined unique Japanese cutoff values for the Chemical Intolerance subscale (40), Symptom Severity subscale (20), and Life Impact subscale (10). The subjects whose scores met or exceeded the cutoff values in any two of the three subscales accounted for $88.4 \%$ of the patient group but only $14.5 \%$ of the control group. Our results suggest that subjects meeting two of the three criteria can be screened as "suspected of having MCS" in Japan. In comparing the above-mentioned Japanese cutoff values with those developed by Miller and Prihoda in the USA [17], we found Chemical Intolerance to have the same cutoff value in Japan and the USA, while the cutoff value for Symptom Severity in Japan was about half that in the USA [17]. These results may be attributable to the frequency distribution of Symptom Severity in MCS patient groups in the USA being limited to within a high score range, while scores of the Japanese MCS patients were widely distributed from low to high. There were no significant differences between the control groups in Japan and the USA.

Another difference from US results was that we did not examine the Other Intolerance subscale in QEESI ${ }^{\odot}$ to evaluate the effects of chemical exposures from foods, etc., because the results of factor analysis for this subscale had previously been reported to be inconsistent with those reported by Miller and Prihoda [17]. Furthermore, ROC analysis also showed low sensitivity (69.9\%), low specificity (54.7\%), and low area under the ROC curve (0.692) in this study. Therefore, we concluded that the Other Intolerance subscale should be excluded when applying the QEESI $^{\odot}$ to evaluate subjective symptoms of MCS patients in Japan. Instead, we used the Life Impact subscale, which had high discrimination ability, the same as that of the Symptom Severity subscale.

We reconfirmed in this study that the $\mathrm{QEESI}^{(}$is effective for screening of Japanese MCS patients, and obtained optimal cutoff values. However, the definition of MCS is still under discussion. Recently, a scientific review of MCS in Australia [1] stated that several primary research needs are evident, including the establishment of agreed diagnostic criteria that are acceptable to clinical and scientific groups. The QEESI ${ }^{\odot}$ is at least useful to screen patients who are suffering from a low level of environmental chemicals such as in MCS and thus to help establish the clinical definition of MCS.

QEESI $^{\odot}$ is also used in other countries such as the USA, Germany, Taiwan, and Korea, so it is an effective means of comparing subjective symptoms among patients in different countries. Moreover, unlike conventional questionnaires which ask only for "yes" or "no" responses, QEESI ${ }^{\odot}$ asks subjects to evaluate their subjective condition on a scale of $0-10$, making it possible to perform certain quantitative analyses as well as effectiveness of treatments, multivariate analysis to clarify clinical condition, etc.

In the future, we will conduct further questionnaire surveys using the QEESI $^{\odot}$, targeting the general population, with randomly selected and distinct groups of MCS as well as patients with other diseases such as sick house syndrome, allergies and mental disorders including depression.

Acknowledgments The authors are grateful to Prof. Yoshiharu Aizawa for his valuable assistance. This study was supported in part by grants-in-aid for scientific research (Hojo C 19510074), JSPS, Japan and a grant-in-aid for scientific research subsidized by the Ministry of Health, Labor, and Welfare and by the Long-range Research Initiative of the Japan Chemical Industry Association.

\section{References}

1. Australian Government: A Scientific Review of Multiple Chemical Sensitivity: Identifying Key Research Needs; Working Draft Report prepared by the National Industrial Chemicals Notification and Assessment Scheme (NICNAS) and the Office of Chemical Safety (OCS), November 2008; p. 1-72. http://www. nicnas.gov.au/Current Issues/MCS ReportPDF.pdf.

2. Ashford NA, Miller CS. Appendix C: environmental exposure and sensitivity inventory. In: Chemical exposure: low levels and high stakes. 2nd ed. New York: Wiley; 1998. p. 371-84.

3. Bailer J, Rist F, Witthoft M, Paul C. Validation of a screening instrument by multiple chemical sensitivity (MCS): the chemical odor sensitivity scale (COSS). Psychother Psychosom Med Psychol. 2004;54(11):396-404.

4. Bailer J, Witthot M, Rist F. The Chemical Odor Sensitivity Scale: reliability and validity of screening instrument for idiopathic environmental intolerance. J Psychosom Res. 2006;61(1):71-9.

5. Bailer J, Witthoft M, Rist F. Psychological predictors of shortand medium term outcome in individuals with idiopathic environmental intolerance (EI) and individuals with somatoform disorder. J Toxicol Environ Health. 2008;71:766-75.

6. Bell IR, Miller CS, Schwartz GE. An olfactory-limbic model of multiple chemical sensitivity syndrome: possible relationships to kindling and affective spectrum disorders. Biol Psychiatry. 1992;32(3):218-42.

7. Caress SM, Steinemann AC. A review of a two-phase population study of multiple chemical sensitivities. Environ Health Perspect. 2003;111(12):1490-7.

8. Cullen MR. The worker with multiple chemical sensitivities: an overview. Occup Med. 1987;2(4):655-61.

9. Meggs WJ, Dunn KA, Bloch RM, Goodman PE, Davidoff AL. Prevalence and nature of allergy and chemical sensitivity in a general population. Arch Environ Health. 1996;51(4):275-82. 
10. Witthoft M, Rist F, Baller J. Abnormalities in cognitive- emotional processing in idiopathic environmental intolerance and somatoform disorders. J Behav Ther Exp Psychiatry. 2008; (Epub ahead of print).

11. Randolff TG. Ecologic orientation in medicine: comprehensive environmental control in diagnosis and therapy. Ann Allergy. 1965;23:11-22.

12. Cullen MR. The worker with multiple chemical sensitivities: an overview. Occup Med. 1987;2:655-61.

13. Nethercott JR, Davidoff LL, Curbow B, Abbey H. Multiple chemical sensitivities syndrome: toward a working case definition. Arch Environ Health. 1993;48:19-26.

14. Anonymous. Consensus on multiple chemical sensitivity. Multiple chemical sensitivity. Arch Environ Health. A 1999 consensus, 1999; 54(3):147-9.

15. Lacour M, Zunder T, Schmidtke K, Vaith P, Scheidt C. Multiple chemical sensitivity syndrome (MCS)—suggestions for an extension of the U.S. MCS - case definition. Int J Hyg Environ Health. 2005;208:141-51.

16. Ishikawa $S$, Miyata $M$, Namba $T$, Nishimoto $H$. Diagnosis of chemical sensitivity. Jpn Med J. 1998;3857:25-9. (in Japanese).

17. Miller CS, Prihoda TJ. The Environmental Exposure and Sensitivity Inventory (EESI): a standardized approach for measuring chemical intolerances for research and clinical applications. Toxicol Ind Health. 1999;15(3-4):370-85.
18. Miller CS, Prihoda TJ. A controlled comparison of symptoms and chemical intolerances reported by Gulf War veterans, implant recipients, and persons with multiple chemical sensitivity. Toxicol Ind Health. 1999;15(3-4):386-97.

19. Ishikawa S, Miyata M. Multiple chemical sensitivity: criteria and test methods for diagnosis (in Japanese). Allergol Immunol. 1999;6(7):990-8. (in Japanese).

20. Hojo S, Kumano H, Yoshino H, Kakuta K, Ishikawa S. Application of Quick Environment Exposure Sensitivity Inventory (QEESI ${ }^{\circledR}$ ) for Japanese population: study of reliability and validity of the questionnaire. Toxicol Ind Health. 2003;19(2-6):41-9.

21. Hojo S, Yoshino H, Kumano H, Kakuta K, Miyata M, Sakabe K, et al. Use of QEESI $^{\odot}$ questionnaire for a screening study in Japan. Toxicol Ind Health. 2005;21(5-6):113-24.

22. Hojo S, Kumano H, Ishikawa S, Miyata M, Sakabe K. Clinical characteristics of physician- diagnosed patients with multiple chemical sensitivity in Japan, Int. J Hyg Environ Health. 2008;211:682-9.

23. Hojo S, Ishikawa S, Kumano H, Miyata M, Mastui T, Sakabe K. Subjective and objective characteristics of patients with multiple chemical sensitivity in Japan. Jpn J Clin Ecol. 2007;16(2):10416.

24. Miller CS, Mitzel HC. Chemical sensitivity attributed to pesticide exposure versus remodeling. Arch Environ Health. 1995;50:11929. 